\title{
Uso inapropiado de psicofármacos prescritos: diazepam intravenoso en la emergencia psiquiátrica.
}

Inappropriate use of prescribed psychotropic drugs: Intravenous diazepam in the psychiatric emergency.

Fabiola Quispe-Turpo 1,2,a, Jéssica Huanco-Condori ${ }^{1,3, a}$, Lizardo Cruzado ${ }^{1,3, b}$.

\section{RESUMEN}

Las benzodiacepinas (BZD) son medicamentos esenciales en el tratamiento de diferentes problemas de salud: en el Petitorio Nacional de Medicamentos de nuestro país figuran cinco de ellas autorizadas para administración oral o parenteral. Al inicio de su uso en la década de 1960, fueron prescritas de forma amplia e irrestricta; el desarrollo posterior de fenómenos de tolerancia y abstinencia desatados por su uso prolongado restringió enérgicamente su empleo, a veces por debajo de las reales necesidades clínicas. Aunque las BZD están incluidas en el grupo IV de sustancias controladas de la Agencia de Control de Drogas (DEA) de los Estados Unidos de América, se acepta que, en general, no son drogas primarias de abuso, y los fenómenos adictivos relacionados con su uso requieren factores adicionales de riesgo tales como comorbilidad con otras drogodependencias. Es necesario distinguir entre casos de "uso inapropiado" de las BZD y aquellos que presentan criterios de franca dependencia. De hecho, se han reportado pocos casos de uso inapropiado de BZD prescritas por vía parenteral. Se presenta y discute el de una mujer de 51 años que, por un tiempo prolongado, acudió a emergencia psiquiátrica debido a crisis de disforia y ansiedad (no de pánico), recibiendo en varias oportunidades inyecciones intravenosas de diazepam, al punto que empezó luego a acudir reiteradamente para exigir la medicación sin que fuera médicamente necesaria. Es conveniente, por lo tanto, que el profesional que prescriba BZD parenterales, lo haga cautelosamente a fin de prevenir un proceso adictivo que puede generar serias consecuencias.

PALABRAS CLAVE: Diazepam; benzodiazepinas; trastornos relacionados con sustancias.

\section{SUMMARY}

Benzodiazepines (BZDs) are essential drugs used in the treatment of different health problems: Peru's National Drug Petitionary lists five of them allowed for oral or parenteral administration. At the beginning in the 1960s, BZDs were widely and unrestrictedly prescribed; the subsequent development of tolerance and withdrawal phenomena, triggered by their prolonged use, led to drastic prescription restrictions, sometimes below the real clinical needs. Although BZDs are included in the Drug Enforcement Administration (DEA) group IV of controlled substances, it is accepted that, in general, they are not primary drugs of abuse, and the development of addictive phenomena only occurs in the presence of comorbidity with other drug addictions. It is necessary to distinguish between the

\footnotetext{
Instituto Nacional de Salud Mental "Honorio Delgado - Hideyo Noguchi”. Lima, Perú.

Facultad de Medicina. Universidad Nacional Mayor de San Marcos. Lima, Perú.

Facultad de Medicina. Universidad Peruana Cayetano Heredia. Lima, Perú.

Médica residente de Psiquiatría.

Psiquiatra.
} 
Uso inapropiado de psicofármacos prescritos: diazepam intravenoso en la emergencia psiquiátrica.

"inappropriate use" or "misuse" of the BZDs and those cases that meet criteria of full dependence. There are few reported cases of inappropriate use of BZD prescribed by parenteral route. The case reported here is one of a 51-years-old woman who, over the course of several years, went to a psychiatric emergency service due to crisis of dysphoria and anxiety (but not a panic attack), and received on several occasions, intravenous injections of diazepam, to the point that later she used to go repeatedly to demand such medication, without being medically necessary. It is, therefore, important for the prescribing professional to use parenteral BZDs cautiously and carefully, in order to prevent an addictive process that can generate serious consequences.

KEYWORDS: Diazepam; benzodiazepines; substance-related disorders.

\section{INTRODUCCIÓN}

A partir de la introducción del clordiazepóxido (1960) y el diazepam (1963), las benzodiacepinas (BZD) han devenido entre los fármacos más empleados en todo el mundo, tanto por médicos generales como por especialistas, para el tratamiento de problemas de insomnio y ansiedad (1). En nuestro país, Lima Metropolitana registra en la población adulta una prevalencia de vida de uso de "tranquilizantes" y "pastillas para dormir" de $0,6 \%$; la prevalencia es similar en adolescentes, en los que la edad de inicio oscila entre los 12 y los 15 años (2). Es muy probable que estas cifras infravaloren los datos poblacionales reales. En los Estados Unidos en el año 2017, por ejemplo, se reporta en individuos mayores de 12 años una prevalencia anual del "mal uso" de tranquilizantes de 2,2\% (aproximadamente 6 millones de personas) pero solo un $0,3 \%$ (739 000 personas) cumplía criterios de abuso o dependencia. Datos similares han sido reportados en otras partes del mundo (3), y se presupone una frecuencia mayor en países de Sudamérica donde las BZD se consiguen fácilmente, sin prescripción médica (4). Además puede apreciarse un incremento progresivo de estas cifras a lo largo de épocas recientes $(3,5)$, todo esto en el contexto de la "medicalización" y "psiquiatrización" de la sociedad, donde la expectativa de una receta farmacológica para paliar todas y cada una de las molestias presentadas por el paciente, aunque no tengan dimensión clínica específica, suelen llevar a la prescripción liberal e irrestricta de medicamentos tranquilizantes $(6,7)$, a lo que se ha denominado "afronte químico" del sufrimiento (8).

Se denomina "uso inapropiado" o "mal uso" de medicamentos al consumo de fármacos por razones distintas a aquellas por las que fueron prescritos, incluyendo variaciones en la dosis y tiempo indicados $(1,9)$. Los medicamentos más involucrados en esta práctica son usualmente aquellos controlados (en
Norteamérica, por orden de frecuencia: los analgésicos opioides, los tranquilizantes e hipnóticos como las BZD, y los estimulantes como las anfetaminas) (10). El Instituto Nacional contra el Abuso de Drogas de los EEUU denomina "uso no medicinal" a estas prácticas y abarca en ellas incluso si una persona usa la medicación de otra (aún por un problema médico legítimo) o toma un medicamento para llegar a un estado de euforia o "high" (10). El uso inapropiado se diferencia del abuso y la dependencia en que este concepto no apunta a fenómenos biológicos subyacentes ni a consecuencias en la funcionalidad del individuo, sino que designa conductas específicas: una persona con dolor neoplásico puede desarrollar, por ejemplo, dependencia física a los opioides (tolerancia y abstinencia) pero no incurrir en uso inapropiado de los fármacos, y a la inversa, muchos individuos hacen mal uso de fármacos, a veces de modo intermitente o esporádico, sin llegar a cumplir criterios de abuso ni de dependencia (9). Obviamente no son conceptos totalmente excluyentes pues todos tienen sustratos biológicos pero las definiciones, a nivel epistémico, se estructuran haciendo énfasis en diferentes aspectos del fenómeno.

No existe una definición de consenso acerca del "uso inapropiado" de medicamentos, en parte a causa de las creencias distintas por parte de profesionales y pacientes sobre lo que es "uso apropiado", y además por la ambivalencia que genera el anhelo médico de ayudar a calmar problemas reales de nuestros pacientes -pese al riesgo de sobreprescribir fármacos-, y a la vez el deseo de evitar crear problemas de abuso o dependencia -pero con riesgo de privar de tratamiento necesario a quien lo requiere realmente- (11). También se ha señalado la escasa o nula formación médica acerca de estos tópicos -lo que perpetúa en los prescriptores la idea sesgada de que el abuso de drogas está fundamentalmente relacionado con drogas ilegales y es cometido por personas marginales-. 
Lamentablemente, el uso indebido de medicamentos prescritos acarrea graves consecuencias pues no solo puede conllevar a abuso y dependencia, sino a intoxicaciones, sobredosis, accidentes y muerte (12). Actualmente en los EEUU se registra una preocupante epidemia de abuso de BZD y opioides, que conlleva elevadas tasas de morbimortalidad (12). Es necesario tomar conciencia de esos riesgos e introducir en nuestro medio la temática del uso inapropiado de medicamentos prescritos, para ponderadamente administrar las BZD sin proscribir su empleo en las dimensiones clínicamente justas, y a la vez que prevenir el uso inapropiado, detectar los posibles casos de este problema prevalente y muchas veces inadvertido.

La literatura sobre "mal uso" de BZD por vía oral es abundante. Sin embargo, hasta donde conocemos hay solo un reporte sobre este tipo de problema asociado a BZD de uso parenteral (13). Esto nos motiva a reportar un caso de nuestra experiencia local y reflexionar con la literatura actualizada al respecto.

\section{Caso clínico}

Mujer de 51 años, instrucción secundaria, divorciada, procedente de Lima, madre de 3 hijos (34, 32 y 13 años de edad). Tiene trabajos eventuales como vendedora. A los 16 años tuvo una consulta psiquiátrica por conducta rebelde, no asistía a clases y prefería ir a fiestas. No siguió tratamiento. A los 28 años de edad acudió a consulta externa de psiquiatría refiriendo molestias de disforia, llanto y agobio emocional ante sinsabores de la vida como discusiones con su pareja, pero no se detectaron criterios de depresión y se le diagnosticó trastorno adaptativo, se prescribió bromazepam 1,5 mg dos veces al día. Sin embargo, no continuó controles regulares.
Alos 30 años de edad acudió a consulta manifestando palpitaciones, sensación de falta de aire y opresión torácica, que al principio se asociaron con "miedo a morir" pero luego fueron secundarias a peleas con su pareja, con crisis de llanto y amenazas suicidas. No tenían un patrón regular de ocurrencia, desaparecían por largos meses, no se asociaban a agorafobia y tampoco había clara ansiedad anticipatoria. Se le diagnosticó trastorno mixto ansioso depresivo y recibió fluoxetina $20 \mathrm{mg}$ y clonazepam 1,5 mg diarios; además de terapia de pareja, pero la asistencia a sus citas programadas fue escasa. Se quejaba de que la fluoxetina le disminuía la libido y reconoció que en situaciones de estrés prefería tomar 4 o 5 pastillas de clonazepam $2 \mathrm{mg}$ para "desconectarse y no pensar". Igualmente, ante el fallecimiento de su madre y un hermano, sufrió exacerbación de sus síntomas en forma de agitación psicomotriz, con autolesiones y descontrol de impulsos (se arañaba y mesaba los cabellos) y amenazas de suicidio que motivaban su atención por emergencia. Fue así que recibía diazepam 10 a $20 \mathrm{mg}$ IV (y en algunas ocasiones se le agregaba clorpromazina $25 \mathrm{mg}$ IM o haloperidol $5 \mathrm{mg} \mathrm{IM}$ ).

Progresivamente sus atenciones en el departamento de emergencia, con características clínicas semejantes a las descritas, fueron más frecuentes. A veces acudía hallándose en estado etílico (nunca se demostró consumo de otras sustancias psicoactivas) y exigía que se le aplique diazepam IV alegando que a ella "siempre le aplicaban eso" o que "solamente eso le hacía bien". Por otro lado, no había registros de que haya sufrido alguna vez distonía aguda ni acatisia por el uso de antipsicóticos.

A lo largo de los últimos 12 años, la paciente tuvo 67 atenciones por emergencia. En todas ellas, cuando no se le aplicaba diazepam IV como ella

Tabla 1. Actitudes sospechosas de "uso inapropiado" de fármacos prescritos*.

- Mayor preocupación por el medicamento que por el problema médico.

- Chantaje emocional: "Usted es el único que puede ayudarme".

- Múltiples síntomas somáticos vagos, exagerados e inespecíficos.

- El usuario tiene conocimiento amplio sobre el medicamento que solicita.

- Actitudes dramáticas, histrioniformes y solicitantes de atención.

- Actitud aduladora al inicio, pero que puede llegar a ejercer presión y hasta veladas amenazas.

- Excusas poco verosímiles y reiterativas: "robaron mi medicina", "perdí mi receta", etc.

- Solicita recetas por gran cantidad de medicamentos; es frecuente la polifarmacia.

- Frecuentan emergencia y no tienen un médico tratante fijo en consulta externa.

*A partir de referencias $(7,9,11)$. 
Uso inapropiado de psicofármacos prescritos: diazepam intravenoso en la emergencia psiquiátrica.

exigía, se tornaba soez y agresiva verbalmente, sin llegar a la agresión física. Por este comportamiento, en 48 ocasiones el personal médico accedió a aplicarle diazepam IV. En las demás, pese a que se le ofrecían medicamentos por vía oral o en combinación (por ejemplo, midazolam $5 \mathrm{mg}$ IM más olanzapina $10 \mathrm{mg}$ VO) para calmar su estado de alteración anímica, llanto e inquietud, los rechazaba y terminaba profiriendo improperios y marchándose. En la última vez que acudió a emergencia, se apreció que mientras aguardaba en la sala de espera, lucía aparentemente calmada. Sin embargo, cuando fue llamada para ser atendida, empezó a respirar más rápido, rechazó responder si mantenía consumo usual de BZD así como desde cuándo no acudía a consulta externa (se atendía supuestamente en otra institución diferente a donde acudía por emergencia). No se le aplicó BZD parenteral, recibió prescripción oral y fue derivada a consultorio externo.

\section{DISCUSIÓN}

La principal motivación que sustenta el uso inapropiado de BZD es enfrentar los síntomas de malestar psicológico asociado o no a problemas de salud mental (14). Se ha descrito distintos patrones de abuso de BZD: a) pacientes que desarrollan algún grado de dependencia física como resultado de una prescripción regular de BZD por un problema subyacente (como ansiedad o insomnio) pero que no llegan a modificar sus dosis prescritas; b) personas que inician una BZD médicamente prescrita pero que luego desarrollan un uso inapropiado e incrementan sus dosis excesivamente; y c) un tercer grupo constituido por personas a las que nunca se les prescribió BZD pero las emplean con fines recreativos o para paliar los efectos de otras drogas consumidas (14). Solo un $2 \%$ de usuarios de BZD pasa del grupo a) al grupo b) (15). Un gran desafío al dirimir entre "mal uso" y "abuso" de BZD es la amplia intersección entre el uso clínico legítimo, la autoadministración con propósitos terapéuticos y el uso no médico (12). De hecho, lo usual es que el uso no prescrito de fármacos controlados se reporte como basado en razones que pretenden ser terapéuticas (12). Se ha señalado asimismo que en esta forma de "afronte químico del sufrimiento" se suscita una tolerancia a los efectos sedativos del fármaco más rápidamente que a los otros efectos terapéuticos específicos, lo que lleva a un escalamiento de dosis con el fin de lograr la sedación deseada con rapidez (8). Los picos de malestar emocional (disputas con familia o amigos, presión laboral o problemas económicos) disminuyen el umbral para crisis emocionales y así gatillan el incremento de dosis de la sustancia controlada, en este caso, la BZD.

Es frecuente que el uso inapropiado de medicamentos se asocie a la presentación de conductas denominadas de "búsqueda de medicamento" ("drug seeking behavior"). En este concepto se enumeran una serie de actitudes sospechosas y que deben motivar una exploración meticulosa para confirmar o descartar dicha posibilidad (tabla 1). Es necesario comprender y asumir que todas estas conductas, aunque incómodas para el médico y muchas veces basadas en el engaño y la mala fe, son sencillamente síntomas propios del cuadro clínico en conjunción con la personalidad de estos pacientes (11). Las conductas de "búsqueda aberrante de medicamentos" son indicadores inespecíficos de diferentes causas potenciales subyacentes: desde problemas de adicción hasta el llamado "chemical coping", es decir, uso de medicamentos para aliviar el sufrimiento de las vicisitudes inevitables de la existencia (15). Un alto porcentaje de individuos con "uso inapropiado" de BZD padece comorbilidad con otro trastorno psiquiátrico (16). Por ello mismo, y de ahí la complejidad del problema, primero debe considerarse la posibilidad de que el paciente realmente tenga síntomas clínicos de fondo, anímicos o no, irresueltos del todo o erróneamente afrontados pues esto podría llevar a la consideración diagnóstica de una "pseudoadicción". En ese sentido, dado el rechazo automático que concita este tipo de etiquetaje diagnóstico, el rol del médico debe ser evaluar objetiva y compasivamente el problema, sin limitarse a reiterar displicentemente la prescripción del fármaco mal usado -lo que solo perpetúa un círculo vicioso-, y más bien tratando de encaminar al paciente hacia un abordaje terapéutico que asuma integralmente todas las aristas del cuadro clínico y de la persona que lo sufre. No debe olvidarse que según el DSM-5 (17), los síntomas de tolerancia y abstinencia que ocurran durante el tratamiento médico con psicofármacos debidamente prescritos (por ejemplo, BZD) no deben tenerse en cuenta para el diagnóstico de un trastorno por uso de sustancias, pues sería totalmente equivocado considerar que estamos ante un problema adictivo en este supuesto caso, si solo son estos criterios los que están presentes. Las personas ansiosas que desarrollan dependencia a BZD (en comparación analógica con las personas diabéticas que requieren insulina) no debieran ser etiquetadas sin más como "adictas a sus medicamentos" (18).

Se ha señalado a los servicios de emergencia psiquiátrica como entornos potencialmente propicios 
para el uso inapropiado de BZD, tanto por parte de pacientes como de médicos prescriptores (19), sobre todo ante crisis emocionales asociadas a personalidad anormal y que podrían manejarse de mejor modo sin recurrir a las BZD. Pese a que poseen menor proclividad al abuso que otros fármacos prescritos como los opioides o los estimulantes, es un hecho que existe una real probabilidad de abuso de las BZD, aunque la prevalencia de dependencia a BZD como sustancia única de abuso es baja (12). Entre las BZD que han mostrado mayor potencial de abuso se encuentra el diazepam (12). Posee una elevada liposolubilidad y por su preferente acceso intravenoso es un candidato ideal para el desarrollo de reforzamiento conductual $\mathrm{y}$, cuando este reforzamiento se desarrolla de manera constante, acarrea un mayor riesgo de perpetuación en el tiempo (20). Tal el caso de nuestra paciente.

¿Cuáles son entonces las indicaciones adecuadas del diazepam intravenoso? Obviamente el veloz inicio de acción del diazepam IV lo hace tentador como recurso de emergencia para la rápida sofocación de crisis de inquietud y agitación psicomotriz, sobre todo cuando no hay sintomatología psicótica. Sin embargo, la debida aplicación de este fármaco debe hacerse en forma diluida y lenta (5 $\mathrm{mg}$ por minuto) (18), a través de una vena periférica adecuada, lo que no siempre es posible en este contexto. Además el uso de diazepam IV acarrea un riesgo de depresión respiratoria que obviamente debe considerarse. Por añadidura, como hemos apuntado, el veloz efecto de "desconexión psíquica inmediata" del diazepam IV lo torna altamente gratificante en casos de reacciones anormales de personalidad, como fue el caso de la paciente. La literatura actual recomienda de manera muy circunscrita el uso de diazepam IV, incluso referencias autorizadas recientes no mencionan al diazepam IV en el uso de crisis de agitación psicomotriz $(21,22)$. Aparte de las indicaciones no psiquiátricas (como relajante muscular, en distonía secundaria a antipsicóticos, o anticonvulsivante), las prescripciones usuales del diazepam IV abarcan actualmente el tratamiento agudo de cuadros de estupor catatónico, estados conversivos-disociativos, crisis de pánico severas, acatisia grave, algunas intoxicaciones por sustancias psicoactivas además de estados de abstinencia alcohólica y delirium tremens en que no se pueda tolerar la vía oral (23).

En el caso de nuestra paciente podemos apreciar cómo la prescripción de BZD desbordó el uso respecto a diagnósticos pretendidamente legítimos (trastorno adaptativo, trastorno mixto ansioso-depresivo) hasta afincarse en condiciones no médicas como la presencia de problemas interpersonales que le generaban disforia y dolor psíquico que ella no podía tolerar y motivaban su exigencia de diazepam IV en atenciones nocturnas por la emergencia psiquiátrica. Es lamentable cómo la actitud desaprensiva o excesivamente complaciente de los médicos prescriptores fue reforzando la conducta anómala de la paciente. Este reporte pretende alertar acerca del uso de BZD IV en situaciones clínicas en que existe alto riesgo de desarrollo de conductas de "uso inapropiado" de BZD, pues la literatura señala que las BZD provocan más que un real fenómeno de dependencia un problema de tipo conductual (24). Actualmente, la perspectiva autorizada es considerar a las BZD como medicamentos muy útiles y seguros, que deben administrarse adecuadamente pues brindan efectos inmediatos que no se alcanzan con otros fármacos y no se debieran soslayar del arsenal psicofarmacológico. Las BZD han sido estigmatizadas, pero es imprescindible recordar que no hay evidencias de que sean mal usadas mientras se prescriban adecuadamente a pacientes que no sean abusadores de otras sustancias psicoactivas ya que el principal problema asociado a su uso es el riesgo de adicción conductual más que una dependencia claramente farmacológica (24). El médico prescriptor debe estar alerta y restringir el uso de BZD IV a casos muy puntuales y discriminados. No debemos privar a nuestros pacientes de medicamentos eficaces y bien tolerados debido a temores inmotivados o ignorancia de la literatura desprejuiciada (25).

\section{Correspondencia:}

\section{Lizardo Cruzado}

Departamento de Emergencia.

Instituto Nacional de Salud Mental "Honorio Delgado - Hideyo Noguchi".

Jr. Eloy Espinoza 709. Urb. Palao. San Martín de Porres.

Lima 15102. Lima, Perú.

Teléfono: (51) 6149205.

Correo electrónico: lizardo.cruzado.d@upch.pe

Conflictos de interés: Los autores declaran no tener conflicto de interés alguno.

Financiamiento: Autofinanciado

\section{REFERENCIAS BIBLIOGRÁFICAS}

1. World Health Organization. Programme on substance abuse. Rational use of benzodiazepines. Copenhagen: WHO; 1996. 
Uso inapropiado de psicofármacos prescritos: diazepam intravenoso en la emergencia psiquiátrica.

2. Instituto Nacional de Salud Mental. Estudio Epidemiológico de Salud Mental en Lima Metropolitana y Callao. Replicación 2012. Informe General. Anales de Salud Mental. 2013; 29(Sup1): $0-0$.

3. Votaw VR, Geyer R, Rieselbach MM, McHugh RK. The epidemiology of benzodiazepine misuse: A systematic review. Drug Alcohol Depend. 2019; 200: 95-114.

4. Dell'osso B, Lader M. Do benzodiazepines still deserve a major role in the treatment of psychiatric disorders? A critical reappraisal. Eur Psychiatry. 2013; 28:7-20.

5. Blanco C, Han B, Jones CM, Johnson K, Compton WM. Prevalence and correlates of benzodiazepine use, misuse, and use disorders among adults in the United States. J Clin Psychiatry. 2018; 79 (6): 12174. doi: $10.4088 / \mathrm{JCP} .18 \mathrm{~m} 12174$

6. Ortiz-Lobo A, Sobrado AM. El malestar que producen los problemas de la vida. AMF. 2013; 9:366-372.

7. Borràs-Vives $\mathrm{R}$, Boada-Loro $\mathrm{S}$, CasamitjanaCucurella N, Casas-Sánchez X, Guayta-Escolies R, Gironella-Ferrer C, et al. Abuso de medicamentos: ¿la adicción del siglo XXI? FMC. 2007; 14:255-262.

8. Weaver M. Prescription sedative misuse and abuse. Yale J Biol Med. 2015; 88:247-256.

9. Isaacson JH, Hopper JA, Alford DP, Parran T. Prescription drug use and abuse. Postgrad Med. 2005; 118: 19-26.

10. National Institute on Drug Abuse; National Institutes of Health; U.S. Department of Health and Human Services. El uso indebido de los medicamentos recetados - Reporte de investigación. Washington DC: National Institute on Drug Abuse; 2020. Disponible en: https://www.drugabuse.gov/es/ publicaciones/los-medicamentos-de-prescripcionabuso-y-adiccion/panorama-general 08/08/2020

11. Parran T. Prescription drug abuse. Med Clin North Am. 1997; 81: 967-978.

12. Nielsen S. Benzodiazepines. En: Nielsen S, Bruno R, Schenk S (eds.). Non-medical and illicit use of psychoactive drugs. Cham: Springer; 2017. pp. 141160.

13. Kaminer Y, Modai I. Parenteral abuse of diazepam: a case report. Drug Alcohol Depend. 1984; 14(1):6365.
14. Liebrenz M, Schneider M, Buadze A, Gehring MT, Dube A, Caflisch C. High-dose benzodiazepine dependence: A qualitative study of patients' perceptions on initiation, reasons for use, and obtainment. Plos One. 2015; 10(11): e0142057.

15. Weaver M. Prescribing medications that have potential for abuse. J Clin Outcomes Manag. 2009; 16: 171-179.

16. Schmitz A. Benzodiazepine use, misuse, and abuse: A review. Ment Health Clin. 2016; 6: 120-126.

17. American Psychiatric Association. Diagnostic and statistical manual of mental disorders. Arlington, VA: American Psychiatric Association; 2013.

18. Stahl SM. Prescriber's guide. $6^{\text {th }}$ ed. London: Cambridge University Press; 2017.

19. Shaner R. Benzodiazepines in psychiatric emergency settings. Psychiatr Ann. 2000; 30: 268-275.

20. Singh RKL, Jain R, Ray R, Gupta YK. Abuse liability of diazepam through different routes. Indian J Physiol Pharmacol. 2001; 45:181-190.

21. Garriga M, Pacchiarotti O, Kasper S, Zeller SL, Allen MH, Vazquez G, et al. Assessment and management of agitation in psychiatry: expert consensus. World J Biol Psychiatry. 2016;17:86-128. doi: 10.3109/15622975.2015.1132007

22. Vieta E, Garriga M, Cardete L, et al. Protocol for the management of psychiatric patients with psychomotor agitation. BMC Psychiatry. 2017; 17:328. doi: 10.1186/s12888-017-1490-0

23. Starcevic V. The reappraisal of benzodiazepines in the treatment of anxiety and related disorders. Expert Rev Neurother. 2014; 14: 1275-1286. doi: 10.1586/14737175.2014.963057

24. de las Cuevas C, Sanz E, de la Fuente J. Benzodiazepines: more "behavioural" addiction than dependence. Psychopharmacology. 2003; 167: 297303. doi: 10.1007/s00213-002-1376-8

25. Balon R, Starcevic V, Silberman E, Cosci F, Dubovsky S, Fava GA, et al. The rise and fall and rise of benzodiazepines: a return of the stigmatized and repressed. Braz J Psychiatry. 2020; 42: 243-244. doi: 10.1590/1516-4446-2019-0773

Recibido: 12/10/2020

Aceptado: 10/05/2021 